\title{
Confier mon autobiographie à un prête-plume?
}

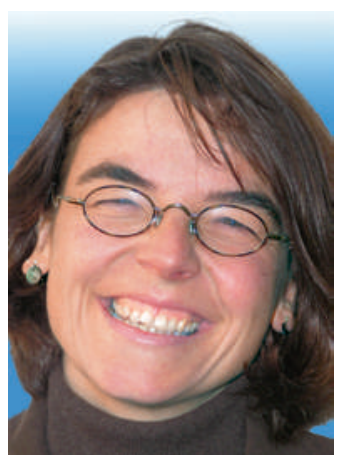

Christina Aus der Au

\section{Références}

- Papassotiropoulos A et al., Human genome-guided identification of memory-modulating drugs. PNAS. Octobre 2013.

- Schapp W. In Geschichten verstrickt. Zum Sein von Mensch und Ding. Frankfurt/ Main; 2004 (orig. 1953)

\section{- Dennett D. The Self as a} Center of Narrative Gravity. In Kessel F, Cole P, Johnson D (éd.). Self and Consciousness: Multiple Perspectives, Hillsdale; 1992.

* PD Christina Aus der $\mathrm{Au}$ docteur en théologie, directrice du Centre de développement ecclésial de la Faculté de théologie de l'université de Zurich, privat-docent en théologie systématique/dogmatique de l'université de Bâle et membre de la rédaction Ethique du Bulletin des médecins suisses.
Le battage médiatique autour du projet du génome humain à peine retombé, voilà qu'un nouveau sujet s'apprête à faire la une! L'information n'est pas encore sortie du cercle des journaux scientifiques, mais elle aurait tout pour susciter de vifs débats dans les commissions d'éthique, les séminaires philosophiques et les rencontres théologiques: à Bâle, des spécialistes des neurosciences sont sur le point de découvrir des possibilités d'effacer des souvenirs négatifs à l'aide de médicaments psychotropes. Par une analyse génétique, ils ont pu identifier les molécules correspondantes, ce qui leur a permis de synthétiser ensuite le médicament adéquat.

Dans l'expérience menée chez des volontaires sains, une seule dose a eu pour effet qu'ils se souvenaient beaucoup moins bien des images négatives qu'on leur avait montrées plus tôt, tandis que leur souvenir des images neutres ou positives restait intact. Ce résultat est du plus haut intérêt pour les personnes souffrant d'états de stress post-traumatique. Cet effet de réduction des mauvais souvenirs pourrait, selon les auteurs, être exploité pour soulager les symptômes tels que cauchemars et flash-back traumatiques chez ces patients.

Un trouble post-traumatique peut faire vivre l'enfer à la personne qui en souffre. La perspective de pouvoir oublier les souvenirs qui la tourmentent est paradisiaque à cet égard. Connaître un nouveau départ, pouvoir revivre, dormir et rêver en faisant table rase du passé - je le leur souhaite du fond du cœur. Ces recherches sont extrêmement importantes pour eux, et au nom de quels prétendus principes moraux celui qui n'aurait pas vécu le même calvaire pourraitil se permettre de les interdire?

Mais comme tant de fois auparavant, ce scénario a été amplement médiatisé par le cinéma. A-t-on le droit de supprimer des souvenirs pénibles de la mémoire? Jusqu'où va la perte de personnalité? Dans le film «Eternal Sunshine of the Spotless Mind» (2004, titre québécois «Du soleil plein la tête»), Jim Carrey et Kate Winslet, un couple déchiré, tentent de s'oublier l'un l'autre en recourant à un procédé d'effacement neuronal. Comme c'est une comédie romantique, le couple se retrouve et l'histoire finit bien, mais le film traite aussi des possibilités d'emploi abusif des souvenirs ainsi volés, de la violation de l'intimité dans ce processus et de l'impossibilité de savoir si l'on peut vraiment vouloir tout cela.

On pourrait poursuivre sur cette idée. Hormis les expériences traumatiques sévères, nous avons presque tous connu dans notre vie des événements et des périodes que nous préférerions oublier, ne serait-ce que les chagrins d'amour.

Seulement voilà - quels souvenirs voudrais-je effacer? Et à quel point doivent-ils disparaître afin que leurs effets ne se fassent vraiment plus sentir? Ma confrontation avec les expériences pénibles et tristes ne me marque-t-elle pas bien au-delà de cette phase? Et qu'arrivera-t-il si je me souviens encore de l'«après», mais pas de ce que j'ai tenté de toutes mes forces de refouler, de compenser ou d'assimiler? Ou si je dois gérer mon «présent» sans savoir ce qui me fait réagir si vivement dans certaines situations?

Quelles parties puis-je supprimer de ma biographie en espérant rester la même? Tout cela ne reposet-il pas sur l'idée que l'être humain se (dé)construit comme un «Lego» - et donc que certains éléments de mon histoire peuvent en être retirés sans compromettre le reste de l'édifice? D'ailleurs, cette conception réductrice qui ne considère que les éléments isolés n'a-t-elle pas déjà été sérieusement remise en question dans d'autres disciplines - en médecine classique par la psychosomatique, pour ne citer que cet exemple, et en physique par la physique quantique, en biologie par l'écologie, en économie par la sociologie, en génétique par l'épigénétique et en éthique par les neurosciences? Je ne parle pas ici d'une relation fumeuse avec une «globalité» ésotérique telle qu'on la voit se répandre dans toutes les disciplines, mais bien de la dimension historique et narrative, qui se veut purement immanente, de l'être humain et de la nature en général. Wilhelm Schapp avait trouvé la formule très évocatrice de l' «écheveau d'histoires».

Tout ce qui est - et c'est particulièrement vrai pour l'être humain - est pris dans un écheveau d'histoires. Et les souvenirs sont les fils de cet écheveau dont est faite notre identité, ici et maintenant.

Pour le spécialiste en sciences cognitives Daniel Dennett, notre soi est le noyau narratif de notre existence, et nous sommes tous des auteurs virtuoses qui nous empressons d'intégrer nos coups durs et nos actions dans un récit autobiographique aussi impressionnant et cohérent que possible.

Qu'adviendra-t-il de mon identité si un prêteplume entreprend de rédiger cette autobiographie à ma place - fût-ce avec mon accord - et d'en effacer des éléments?

Christina Aus der $A u^{*}$ 